\title{
Hyperintensities of the anterior horn cells on MRI due to poliomyelitis
}

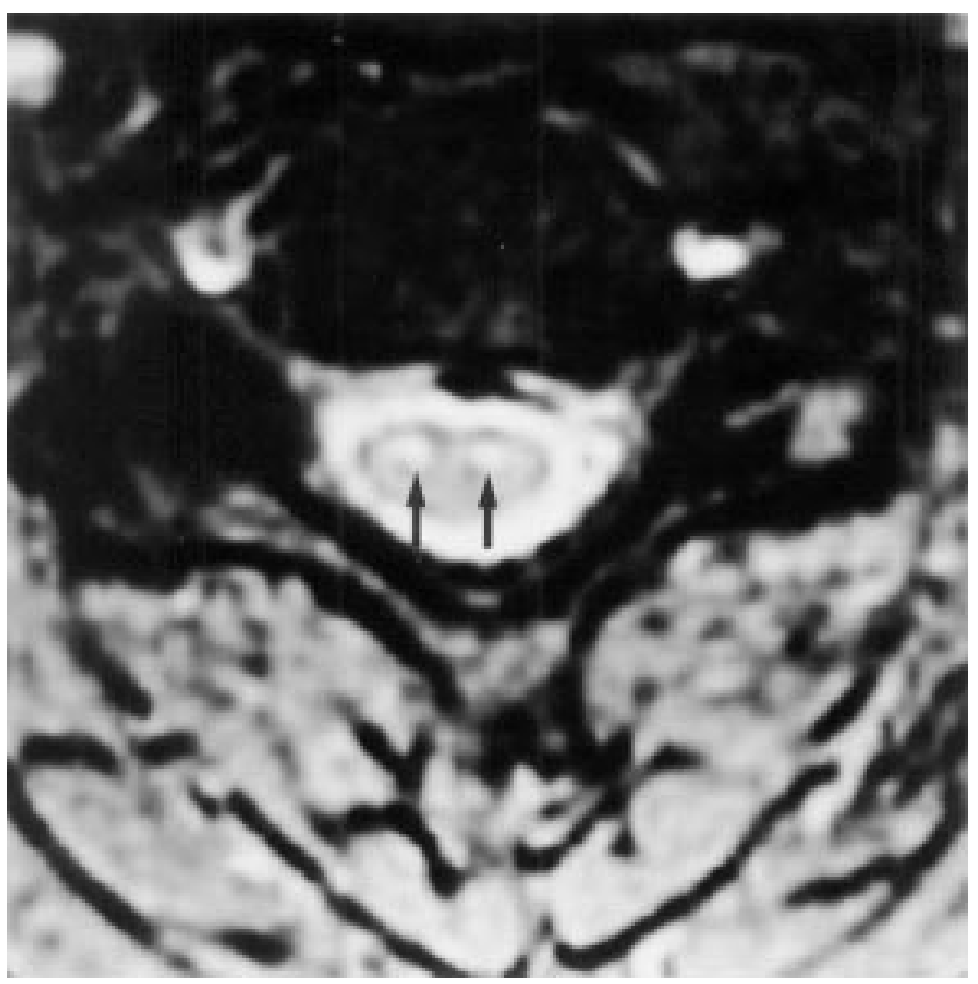

A 63 year old man was seen in the outpatient clinic with a history of recent (nine months) worsening weakness of both arms. At the age of three he had had poliomyelitis involving both shoulder girdles and arms.

His deltoid, biceps brachii, brachialis, triceps brachii, supraspinatus, infraspinatus, and pectoralis major muscles were bilaterally wasted and weak. Deep tendon reflexes were absent in the upper limbs and were normally elicited in the lower limbs. Plantars were flexor and sensory examination was normal.

Electrophysiological studies were suggestive of neurogenic lesion without any evidence of active denervation. MRI of the cervical spine showed high signals in the region of anterior horn cells ( marked with two black arrows) from C4-C6 levels on the T2 weighted images due to sclerosis. The recent worsening was attributed to postpolio syndrome. 